\title{
Entire Phonon Spectrum of Molecular Crystals by the Localized Exciton Sideband Method: Naphthalene*
}

\author{
Raoul Kopelman, Frederick W. Ochs, and Paras N. Prasad \\ Department of Chemistry, The University of Michigan, Ann Arbor, Michigan 48104
}

(Received 24 July 1972)

\begin{abstract}
The complete one-phonon density-of-states of a molecular crystal can be mapped out by a carefully chosen exciton sideband involving a localized molecular internal excitation in a dilute isotopic mixed crystal. A theoretical derivation shows that the necessary criteria are: absence of localized in-band phonons; applicability of a phonon amalgamation limit for the mixed crystal; weak exciton-phonon, guest-lattice coupling and weak guest-host exciton interaction. Experimentally, at least two exciton-phonon sidebands should be investigated, so as to exclude trap and defect transitions, and at least one of the guest transitions should belong to the deep-trap limit and have sidebands weak enough to minimize multiphonon transitions. The above is demonstrated for the naphthalene crystal by high-resolution fluorescence and phosphorescence spectra of $0.25 \% \mathrm{C}_{10} \mathrm{H}_{8}$ in $\mathrm{C}_{10} \mathrm{D}_{8}$ and by phosphorescence of $0.14 \% 2-\mathrm{DC}_{10} \mathrm{H}_{7}$ in $\mathrm{C}_{10} \mathrm{D}_{8}$ at $2^{\circ} \mathrm{K}$. The phosphorescence phonon sidebands correlate surprisingly well with Pawley's "atom-atom" calculated phonon density-of-states, and seems to give a much better phonon density map than the very recent high-resolution inelastic, incoherent neutron scattering data. New Raman data are also presented, showing defect (isotopic impurity) induced bands, which are interpreted in terms of the above phonon density-of-states peaks. Applications to reversible and irreversible thermodynamics are mentioned.
\end{abstract}

\section{INTRODUCTION}

Lattice dynamics are usually investigated experimentally by far-infrared and Raman spectroscopy and by inelastic neutron scattering. ${ }^{1}$ While the above spectroscopic methods reveal the nature of only $\mathbf{k}=0$ modes, inelastic neutron scattering can, in principle, provide information about the phonon spectrum of the entire Brillouin zone. In practice, however, the neutron scattering method has limitations imposed mainly by experimental difficulties. The most informative approach to lattice dynamics is by neutron coherent scattering study. ${ }^{2,3}$ But because of the difficulties due to long and tedious experimentation as well as due to the complexity of organic molecular solids, only few studies of this kind have been made ${ }^{4-6}$ and these are limited to isotropic or uniaxial crystals with one molecule per primitive cell or are incomplete. In recent years the trend has been to conduct a neutron incoherent scattering study which uses simpler experimentation and little computation. The spectrum obtained by the neutron-incoherent scattering has been shown ${ }^{7,8}$ to represent approximately hydrogen amplitude weighted density-of-phonon-states. This is based on the fact that protons act as the predominant scatterers for the neutrons. The incoherent scattering can, thus, at best furnish direct information only about the peaks (or singularities) in the total density-of-phonon states. Though the incoherent scattering experiments are simpler than the coherent scattering experiments, still they involve much more sophistication than spectroscopic experiments. Also, the results obtained by the neutron scattering have a larger energy uncertainty compared to the uncertainty in the spectroscopic experiments, require larger samples, and may also be more susceptible to multiphonon processes. In view of this, it would be desirable to design an optical (spectroscopic) experiment which could yield the same information. In this paper we suggest a method which uses optical spectroscopy to map the singularities in the density-of-phonon states. We show that the phonon sidebands in the electronic (or vibronic) emission spectrum (at very low temperatures) corresponding to the guest electronic (or vibronic) transition in a dilute isotopic mixed crystal can give the singularities (including peaks) of the density-of-phonon states of the host. Against these, theoretical calculation can be calibrated.

In some cases, the defects which, invariably, are present in organic solids may be in sufficient concentration to relax the $\mathbf{k}=\mathbf{0}$ selection rule. In such cases one may see some of the density-of-states peaks (which do not correspond to $\mathbf{k}=\mathbf{0}$ modes) in the Raman and/or far-infrared spectra of neat crystals. This well-known method is shown via some Raman spectra to be much inferior as a phonon density mapping.

We present results from the fluorescence and phosphorescence spectra of naphthalene- $h_{8}$ and $2-\mathrm{DC}_{10} \mathrm{H}_{7}$ in naphthalene- $d_{8}$. The peaks of the density-of-phonon states obtained by this method show a remarkable resemblance to the ones obtained by a semiempirical (atom-atom) calculation by Pawley, ${ }^{9,7}$ and seem to give a more faithful representation of the one-phonon spectrum than do the most recent neutron incoherent scattering experiments. ${ }^{8}$ This opens the door for the utilization of such data for both reversible thermodynamics, like heat capacity, and irreversible processes, like radiationless transitions and photochemistry in condensed phases.

\section{THEORY}

The discovery of phonon transitions accompanying the electronic transitions goes back to as early as the 
1950's, and since then a great number of experimental results on phonon side bands have been reported..$^{10,11}$ No serious attempt was made in the past to provide a theoretical understanding of these phonon participations. Only recently have formal theories of electron (exciton)-phonon interaction in molecular solids been developed by Davydov, ${ }^{12}$ Fischer and Rice, ${ }^{13}$ Grover and Silbey, ${ }^{14}$ and Mavroyannis. ${ }^{15}$ Hochstrasser and Prasad $^{16}$ made an effort to bridge the gap that still existed between the theory and the experiments by relating the theoretical findings with experimental observations. Their results show that the phonon side band in the neat crystal spectrum is attenuated when the exciton is in a delocalized state (when the exciton bandwidth is large) and is more pronounced as the exciton bandwidth becomes smaller. This exciton bandwidth effect can be avoided by using a dilute chemically mixed crystal, where the guest-host energy separation is large enough to split the guest level from the host exciton band. However, in the chemically mixed crystal there is a local lattice distortion around the guest site which arises because of the perturbation due to the guest (change in mass coefficient, force constant) and the eigenvectors are different from the delocalized eigenvectors of the neat host lattice, so that some phonons have large amplitudes at the guest site. These are localized or pseudolocalized phonons. Such local modes are expected to dominate the phonon sideband spectrum of the guest transition, thus masking the effect of host (delocalized) phonon bands. This was found to be the case with the systems experimentally investigated by Hochstrasser and Prasad ${ }^{16}$ where the phonon sideband in the guest transition was found to be very different from the phonon sideband of the host neat crystal spectrum. The existence and importance of the pseudolocalized phonons in mixed crystal transitions is very clearly revealed by the work of Chereson, Friedman and Kopelman, ${ }^{17}$ where the phonon sideband in the guest spectrum was found to be very sensitive to isotopic substitution of the guest but not necessarily to that of the host. In view of these facts it is clear that the chemically mixed crystal system is not at all suitable for studying the host phonon bands. Let us examine now the case of a dilute isotopic mixed crystal. Except for a few cases the separation between the electronic protoguest level and the perdeuterohost level is such that the electronic protoguest level exists below the perdeutero exciton band. For this situation we again are "getting rid" of the exciton bandwidth effect on phonon sidebands. The question arises whether localized phonons and pseudolocalized phonons exist in isotopic mixed crystals. Theoretically, the mass defects are small and the expected force constant "defects" are negligible. The recent Raman studies ${ }^{18}$ of the lattice modes in isotopic mixed naphthalene crystals indeed rule out the existence of such local modes and suggest that the phonon states in isotopic mixed crystals are in the amalgamation limit ${ }^{19,20}$ (with very small lattice distortions), that is, the phonon spectrum of the host shows an approximately linear frequency shift with guest concentration while the basic features of the spectrum remain unchanged. The phonon sidebands in the electronic transition of the dilute protoguest, thus, represent the host phonon bands.

At this stage we would like to derive an expression for the phonon sideband in isotopic mixed crystals following the theoretical treatment by Hochstrasser and Prasad. ${ }^{16}$ We start with a real crystal (monoclinic) at a temperature close to absolute zero. The assumption of zero temperature is made for simplicity, besides the fact that this assumption represents approximately our experimental condition (temperature $\sim 1.9-2.0^{\circ} \mathrm{K}$ ). We further assume that the guest is situated at a lattice site $n \alpha=o \alpha$ where $n$ labels the unit cell and $\alpha$ is the site (interchange equivalent) index in the unit cell. The unit cell where the guest is situated represents our origin of the coordinate system.

The Hamiltonian for the guest is given as

$$
H_{G}=H_{0}+H_{\text {int }} \text {, }
$$

where

$$
H_{0}=\sum_{f}\left(\Delta E_{f}+V_{f, f}\right) A_{f}^{+} A_{f}
$$

and

$$
H_{\text {int }}=\sum_{n, \alpha, u}\left(\partial V_{f, f} / \partial R_{u n \alpha}\right)_{0} R_{u n \alpha} A_{f}+A_{f} .
$$

Here we have retained only those terms of the full Hamiltonian for the mixed crystals which are important in relation to phonon sidebands. The index $f$ labels the electronic states of the guest. $R_{u n \alpha}$ represents the instantaneous displacement along the $u$ th coordinate of the molecule at site $\alpha$ in the unit cell $n$. The index $u$ assumes six values: three corresponding to translations along $x, y$, and $z$, while the remaining three correspond to the librations, for a semirigid nonlinear molecule. $A_{f}{ }^{+}$and $A_{f}$ are, respectively, the creation and annihilation operators corresponding to the guest electronic state $f . V_{f, f}$ is the solvent shift given as

$$
V_{f f}=\left\langle f\left|\sum_{n \alpha} V_{n \alpha}\right| f\right\rangle-\left\langle g\left|\sum_{n \alpha} V_{n \alpha}\right| g\right\rangle
$$

and is the change in van der Waals interactions due to the transition $g \leftrightarrow f$ of the guest. $H_{\text {int }}$ represents only the linear electron-phonon coupling arising from the change in the equilibrium position of the guest molecule in the host lattice during the $g \leftrightarrow f$ transition of the guest molecule. This change in the equilibrium position of the guest during its electronic transition is a measure of the guest-lattice coupling. $\Delta E_{f}$ is the gas phase guest transition.

As we have mentioned earlier, the phonon spectrum of the isotopic mixed crystal represents the amalgamation limit. In this limit we can express the displacement operators in terms of the delocalized phonon creation and annihilation operators $b_{S, q}{ }^{+}$and $b_{S, q}$, respectively, 
by the following relation ${ }^{21}$ :

$$
\begin{aligned}
R_{u n \alpha}=\sum_{S, q}\left(2 N M \omega_{S, q}\right)^{-1 / 2} \eta_{\alpha}{ }^{u}(S, q) & \\
& \quad \times \exp \left(i \mathbf{q} \cdot \mathbf{x}_{n}\right)\left[b_{S, q}+b_{S,-q}{ }^{+}\right]
\end{aligned}
$$

This is expressed taking $\hbar=1$; $\omega_{S, q}$ is the frequency, $N$ the number of cells, $\mathbf{x}_{n}$ is the position vector of the $n$th unit cell, $q$ is a vector in the Brillouin zone, and $S$ labels the different phonon branches. In the naphthalene crystal (monoclinic with two molecules per primitive cell) there are twelve branches. The branch vectors $\eta_{\alpha}{ }^{u}(S, q)$ are normalized by the following relation:

$$
\sum_{\alpha, u, u u^{\prime}} \eta_{\alpha^{u}}{ }^{\prime}(S, q) m_{u u^{\prime}, \alpha} \eta_{\alpha}{ }^{u^{\prime}}(S, q)=M \delta_{S S^{\prime}}
$$

Here $m_{u u^{\prime}, \alpha}$ is the mass coefficient for the molecule at site $\alpha$ and corresponds to the coordinates $u$ and $u^{\prime}$. In a pure crystal $m_{u u^{\prime}, \alpha}$ for translation is given as

$$
m_{u u^{\prime}, \alpha}=m_{u u^{\prime}} \delta_{u u^{\prime}} \text {. }
$$

This represents the mass of a molecule and is independent of the site index $\alpha$. For librational motion $m_{u u^{\prime}, \alpha}$ represents the moment of inertia and is not necessarily diagonal $\left(u \neq u^{\prime}\right)$ and also it may not be independent of the site index $\alpha$. In an isotopic mixed crystal $m_{u u^{\prime}, \alpha}$ represents an effective mass coefficient at site $\alpha$ which, in principle, can be determined by a selfconsistent calculation. With the use of the Eq. (4) we can express the interaction Hamiltonian of Eq. (2) in terms of the phonon creation and annihilation operators:

$$
\begin{aligned}
H_{\text {int }}=\sum_{l, \beta, u}^{\prime}\left[\left(\partial V_{\left.o \alpha, l \beta^{f, f} / \partial R_{u o \alpha}\right)_{0} R_{u o \alpha}}\right.\right. & \\
& +\left(\partial V_{\left.\left.o \alpha, l \beta^{f, f} / \partial R_{u l \beta}\right)_{0} R_{u l \beta}\right] A_{f}+A_{f}}\right.
\end{aligned}
$$

or

$$
\begin{gathered}
H_{\mathrm{int}}=\sum_{S, q}\left(2 M N \omega_{S, q}\right)^{-1 / 2} L_{S, q}^{f}\left[b_{S, q}+b_{S,-q^{+}}\right] A_{f}^{+} A_{f}, \\
H_{\mathrm{int}}=N^{-1 / 2} \sum_{S, q} D_{S, q}^{f}\left[b_{S, q}+b_{S,-q}+\right] A_{f}^{+} A_{f},
\end{gathered}
$$

where

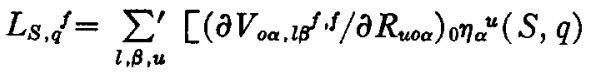

$$
\begin{aligned}
& \left.+\left(\partial V_{o \alpha, l} l \delta, \delta / \partial R_{u l \beta}\right)_{0} \eta_{\beta}{ }^{u}(S, q) \exp \left(i \mathbf{q} \cdot \mathbf{x}_{l}\right)\right],
\end{aligned}
$$

and

$$
D_{S, q}{ }^{f}=\left(2 M \omega_{S, q}\right)^{-1 / 2} L_{S, q}{ }^{f}
$$

In these equations the index $l \beta$ runs over the neighbors of the guest molecule $o \alpha$. We make a canonical transformation of the Hamiltonian (1) to a new Hamiltonian, $\widetilde{H}_{G}=\exp (-\tilde{\lambda}) H_{G} \exp (\tilde{\lambda})$, which has no offdiagonal term linear in $H_{\text {int }} . \tilde{\lambda}$ is then given as

$$
\begin{aligned}
& \left\langle f, 1_{S, q}|\tilde{\lambda}| f, 0_{S, q}\right\rangle \\
& \quad=N^{-1 / 2}\left(D_{S, q}^{f}\left[b_{S, q}+b_{S,-q}^{+}\right] / \omega_{S, q}\right) .
\end{aligned}
$$

Here we have taken the matrix elements only over the electronic state operators $A_{f}{ }^{+}$and $A_{f}$. The phonon Franck-Condon factor for a one-phonon transition is given $\mathbf{a s}^{16}$

$$
\begin{aligned}
\left|\left\langle\Phi_{f, q, S^{1}} \mid \Phi_{g, q, S^{0}}\right\rangle\right|^{2}= & N^{-1}\left(D_{S, q}{ }^{f} / \omega_{S, q}\right)^{2} \\
& \times \exp \left[-N^{-1}\left(D_{S, q}{ }^{f} / \omega_{S, q}\right)^{2}\right]
\end{aligned}
$$

(see Appendix of Ref. 12). The one-phonon intensity is given $\mathbf{a s}^{16}$

$$
\begin{aligned}
I_{\mathbf{1}}^{f}(\omega)= & C\left|\mu_{g f}\right|^{2} \exp \left[-N^{-1} \sum_{S, q}\left(D_{S, q}^{f} / \omega_{S, q}\right)^{2}\right] \sum_{S, q} N^{-1} \\
& \times\left(D_{S, q} f / \omega_{S, q}\right)^{2} \delta\left(\omega-\omega_{S, q}\right) \\
= & I_{0}^{f}(2 N M)^{-1} \sum_{S, q}\left[\left(L_{S, q}\right)^{2} /\left(\omega_{S, q}\right)^{3}\right] \delta\left(\omega-\omega_{S, q}\right) \\
= & I_{\sigma^{f}} G_{o p} f(\omega),
\end{aligned}
$$

where $\mu_{g f}$ is the transition dipole, $C$ is a constant and

$$
G_{o p}{ }^{f}(\omega)=(12 N)^{-1} \sum_{S, q} C_{S, q}{ }^{f} \delta\left(\omega-\omega_{S, q}\right),
$$

where

$$
C_{S, q}=(6 / M)\left[\left(L_{S, q}^{f}\right)^{2} /\left(\omega_{S, q}\right)^{3}\right]
$$

Also,

$$
I_{0}^{f}=C\left|\mu_{o f}\right|^{2} \exp \left\{-N^{-1} \sum_{S, q}\left[\left(D_{S, q}\right)^{2} /\left(\omega_{S, q}\right)^{2}\right]\right\}
$$

measures the total intensity of the zero-phonon line in the $g \leftrightarrow f$ electronic transition of the guest. The one-phonon sideband in the $g \leftrightarrow f$ transition of the guest measures the frequency dispersion of the function $G_{o p} f(\omega)$. The density-of-phonon states for our monoclinic crystal is given as

$$
g(\omega)=(12 N)^{-1} \sum_{S, q} \delta\left(\omega-\omega_{S, q}\right) .
$$

Comparing Eqs. (13) and (16) we see that $G_{o p} f(\omega)$ represents an amplitude weighted phonon density-ofstates. This amplitude is $C_{S, q}{ }^{f}$. Hochstrasser and Prasad $^{16}$ have worked out the dependence of $C_{S, q}{ }^{f}$ on $\omega_{S, q}$ for a cubic crystal with one molecule per unit cell. Their result shows that for acoustic phonons $C_{S, q}{ }^{f} \alpha\left(\omega_{S, q^{-1}}\right)$ whereas for librational optical phonons $C_{S, q}{ }^{f} \alpha\left(\omega_{S, q}{ }^{-1}\right)^{3}$. For the real crystal these two values may represent the lower and upper limits of the dependence of $C_{s, q}{ }^{f}$ on $\omega_{S, q}$. In view of this the amplitude $C_{S, q}{ }^{f}$ is not expected to have singularities and the singularities of $G_{o p}{ }^{f}(\omega)$ should represent the singularities of $g(\omega)$.

So far we have considered only a one-phonon process. In the presence of multiphonon processes the situation is complicated. However, as most organic molecular crystals represent weakly interacting systems, with van der Waals forces holding the molecules together, the guest-lattice coupling is small. For this reason the multiphonon transition probabilities are rather small 


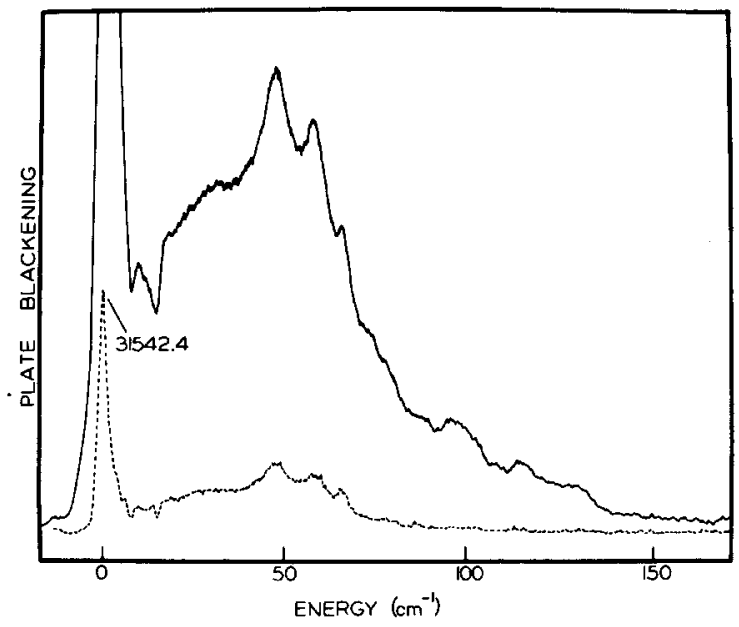

FIG. 1. Densitometer tracing of the fluorescence spectrum of $0.25 \% \mathrm{C}_{10} \mathrm{H}_{8}$ in $\mathrm{C}_{10} \mathrm{D}_{8}$ mixed crystal at $2^{\circ} \mathrm{K}$ and a resolution of $1 \mathrm{~cm}^{-1}$. Dotted spectrum shown to indicate position of zero phonon line and relative intensity of phonon sidebands. $\mathrm{Re}-$ absorption of zero phonon line is significant (see text).

and usually the one-phonon transitions are expected to dominate the phonon sideband. This can also be assured by measuring the relative total intensity of the zero-phonon line compared to that of the phonon sideband.

We thus see that the phonon sideband on the electronic transition of the guest should map the singularities of the density-of-phonon states.

Because of the interference of perdeuterohost absorption with the protoguest absorption it is desirable to study the sideband in the emission spectrum of the guest. However, such a study is complicated by the presence of trap emissions, which may give an impression of being another phonon peak. Such errors can be eliminated, however, by comparing the phonon sideband in the fluorescence with that in the phosphorescence of the guest. When one-phonon transitions dominate the phonon side-band the amplitudes $C_{S, q}{ }^{f}$ should show only small variations for two emissions of the same orbital nature (both $\Pi-\Pi^{*}$ or $\left.n-\Pi^{*}\right)$. As a result, the intensity of the phonon peaks may be slightly different in the two emissions but the prominent spectral features of the phonon sideband should remain unchanged. On the other hand, the transitions due to traps should be very different in these two emissions. On the basis of the above theoretical discussion we suggest that the dilute isotopic mixed crystal seems to be a very suitable system for studying the density-of-phonon states of the host, provided one uses as criterion the simultaneous observation of phonon sidebands in both fluorescence and phosphorescence, or in any two emission spectra. Some complications in the naphthalene fluorescence, due to guest-host exciton coupling, are discussed in the Appendix.

\section{EXPERIMENTAL}

The naphthalene- $d_{8}$ used in the experiments was purchased from Thompson-Packard, Inc. (Little Falls, N.J.), and had an isotopic purity of $99.0 \pm 0.1$ at $\%$ deuterium as determined by mass spectral analysis. The sample was purified by zone refining, then performing a potassium fusion followed by further extensive zone refining. The naphthalene- $h_{8}$ was purchased from James Hinton and had a stated purity of 99.99\%. The naphthalene-2- $d_{1}$ was purchased from Merck, Sharp, and Dohme and had an isotope purity of $98.5 \%$ deuterium. The $\mathrm{C}_{10} \mathrm{H}_{8}$ and $2-\mathrm{DC}_{10} \mathrm{H}_{7}$ were used without further purification. The mixed crystals of composition $0.25 \% \mathrm{C}_{10} \mathrm{H}_{8}$ in $\mathrm{C}_{10} \mathrm{D}_{8}$ and $0.14 \% 2-\mathrm{DC}_{10} \mathrm{H}_{7}$ in $\mathrm{C}_{10} \mathrm{D}_{8}$ were grown from the melt in a Bridgman furnace. The crystal was polished on the cleavage (ab) face to a thickness of approximately $1 \mathrm{~mm}$. The crystal was immersed in pumped liquid helium $\left(2^{\circ} \mathrm{K}\right)$, in a cryostat that had no liquid nitrogen in the optical path.

The fluorescence was excited by front surface illumination with a $1600 \mathrm{~W}$ xenon lamp, using a $110 \mathrm{~g} /$ liter $\mathrm{NiSO}_{4}+60 \mathrm{~g} /$ liter $\mathrm{CoSO}_{4}$ solution in a $10 \mathrm{~cm}$ cell and a $40 \mathrm{mg} /$ liter solution of 2,7-dimethyl-3,6-diazacyclohepta-1,6-diene perchlorate in a $5 \mathrm{~cm}$ cell as filters. For phosphorescence a Corning 7-54 filter was used in place of the perchlorate. The spectra were taken on a Jarrel-Ash model 25-100 1-m-double-Czerny-Turner spectrograph-spectrometer. The fluorescence spectrum was photographed in second order on Kodak SA-1 film. The phosphorescence was photographed in the first order on Kodak $103 a-F$ film with an additional Corning $\mathrm{O}-52$ filter placed before the slits. The

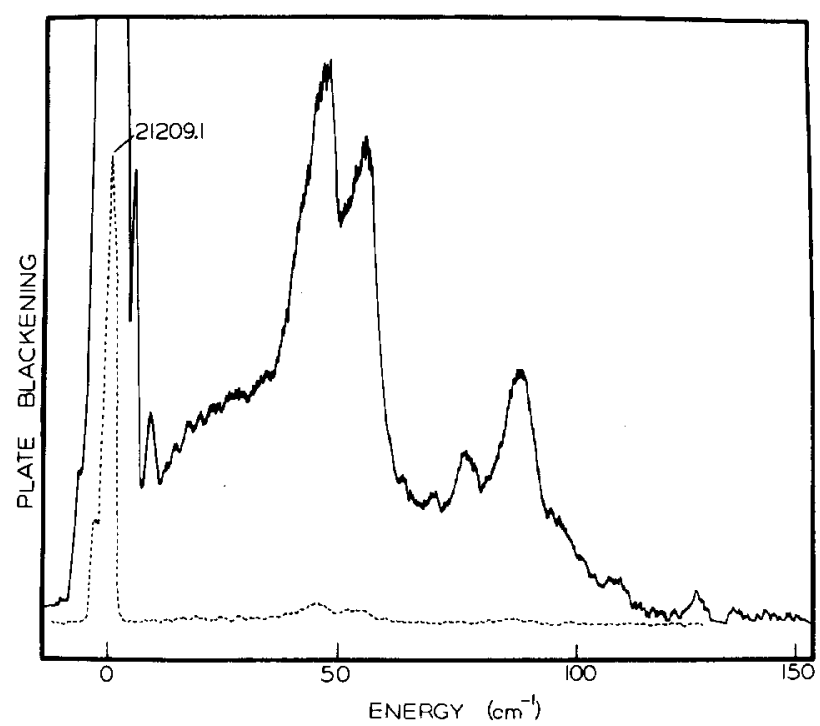

FIG. 2. Densitometer tracing of the phosphorescence of $0.25 \%$ $\mathrm{C}_{10} \mathrm{H}_{8}$ in $\mathrm{C}_{10} \mathrm{D}_{8}$ mixed crystal at $2^{\circ} \mathrm{K}$. Dotted spectrum shown to indicate position of zero phonon line and relative intensity of phonon sidebands. Feature to slightly higher energy is due to phosphorescence of ${ }^{13} \mathrm{C}^{12} \mathrm{C}_{9} \mathrm{H}_{8}$. Sharp feature at about $-5 \mathrm{~cm}^{-1}$ is due to defect phosphorescence. 
TABLE I. Naphthalene phonon peaks $\left(\mathrm{cm}^{-1}\right)$.

\begin{tabular}{|c|c|c|c|c|c|}
\hline $\begin{array}{c}\mathrm{C}_{10} \mathrm{H}_{8} \text { in } \mathrm{C}_{10} \mathrm{D}_{8} \\
\text { Fluorescence }\left(2^{\circ} \mathrm{K}\right)\end{array}$ & $\begin{array}{c}\mathrm{C}_{10} \mathrm{H}_{8} \text { in } \mathrm{C}_{10} \mathrm{D}_{\mathrm{g}} \\
\text { Phosphorescence }\left(2^{\circ} \mathrm{K}\right)\end{array}$ & $\begin{array}{c}2-\mathrm{DC}_{10} \mathrm{H}_{7} \text { in } \mathrm{C}_{10} \mathrm{D}_{8} \\
\text { Phosphorescence }\left(2^{\circ} \mathrm{K}\right)\end{array}$ & $\begin{array}{l}\text { Neutron incoherent } \\
\text { scattering }\left(120^{\circ} \mathrm{K}\right)\end{array}$ & $\begin{array}{c}\operatorname{Raman}^{\mathrm{b}} \\
\mathrm{C}_{10} \mathrm{D}_{8}\left(4^{\circ} \mathrm{K}\right)\end{array}$ & $\begin{array}{l}\text { Theoretical } \\
\mathrm{C}_{10} \mathrm{H}_{8}\end{array}$ \\
\hline $10 \pm 2$ & $8 \pm 1$ & $8 \pm 1$ & & & $8 ?$ \\
\hline $33 \pm 3 d$ & $29 \pm 3^{d}$ & $27 \pm 3^{d}$ & $31 \pm 1$ & & $25 ?$ \\
\hline $47 \pm 1$ & $47 \pm 1$ & $46 \pm 1$ & $39 \pm 1$ & & 38 \\
\hline $58 \pm 1$ & $56 \pm 1$ & $56 \pm 1$ & $54 \pm 2$ & 54 & 49 \\
\hline \multirow[t]{2}{*}{$66 \pm 1$} & $64 \pm 2$ & $64 \pm 2$ & & 64 & $68 ?$ \\
\hline & $70 \pm 2$ & $71 \pm 2$ & & & \\
\hline $77 \pm 2$ & $78 \pm 1$ & $79 \pm 1$ & & 79 & 78 \\
\hline $90 \pm 2$ & $89 \pm 1$ & $89 \pm 1$ & & 84 & 83 \\
\hline $97 \pm 2$ & $96 \pm 2$ & $96 \pm 2$ & $96 \pm 2$ & & \\
\hline $114 \pm 2$ & $111 \pm 3$ & $108 \pm 3$ & & 112 & \\
\hline $129 \pm 3$ & $128 \pm 1$ & $125 \pm 3$ & $120 \pm 3$ & 130 & 127 \\
\hline
\end{tabular}

- Reference 8. Note that these data are for $\mathrm{C}_{10} \mathrm{H}_{8}$.

${ }^{b} \mathbf{k}=0$ modes after M. Ito, M. Suzuki, and T. Yokoyama in Ref. 24, p. 1. Note that a similar comparison of these data with $\mathrm{C}_{10} \mathrm{H}_{8}$ in $\mathrm{C}_{10} \mathrm{D}_{8}$ emission at $4^{\circ} \mathrm{K}$ has been made by D. M. Hanson, J. Chem. Phys. 51, 5063 (1969).

- References 7 and 9. Note that assigning peak values to Pawley's data is arbitrary in view of the fluctuations in his curve, especially above $90 \mathrm{~cm}^{-1}$. The $C_{10} D_{8}$ values seem to be a few percent lower, but we did not list them, as Fig. 2 of Ref. 7 is much smaller than Fig. 1.

d The uncertainty is related to the bandwidth.

effective spectral resolution for both emissions was $1 \mathrm{~cm}^{-1}$.

For the Raman investigation single crystals were grown in a capillary under vacuum. The capillary, containing an optically single crystal, was used as is. It was cooled by a flow of nitrogen vapor which provided a temperature of about $100^{\circ} \mathrm{K}$. The Raman spectra were recorded photoelectrically on a Spex Ramalog double spectrometer at a resolution of 1

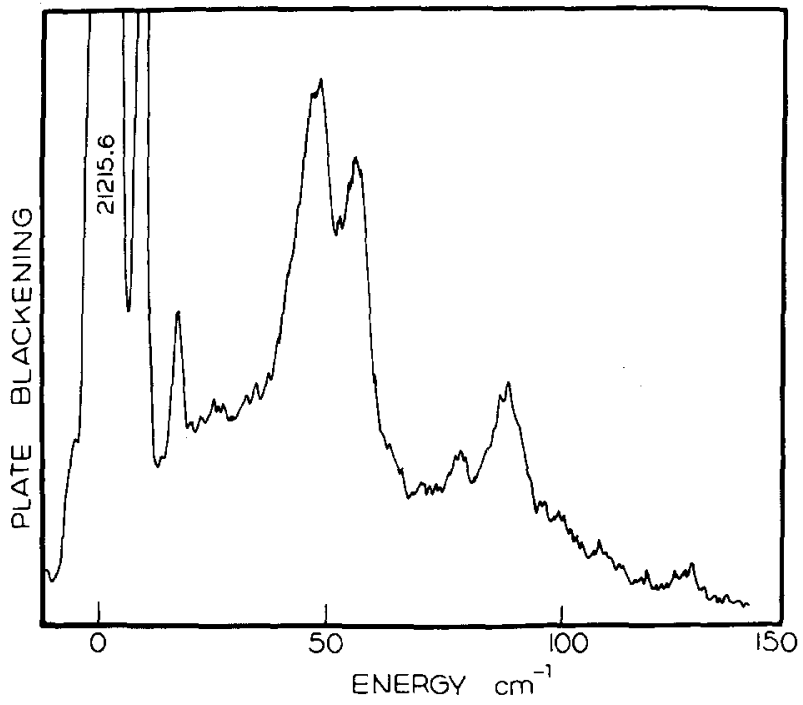

FIG. 3. Densitometer tracing of $2^{\circ} \mathrm{K}$ phosphorescence of $0.14 \% 2-\mathrm{DC}_{10} \mathrm{H}_{7}$ in $\mathrm{C}_{10} \mathrm{D}_{8}$ mixed crystal. Feature to slightly higher energy is due to phosphorescence from $2-\mathrm{D}^{13} \mathrm{C}^{12} \mathrm{C}_{9} \mathrm{H}_{7}$. Feature at $-8 \mathrm{~cm}^{-1}$ is due to $\mathrm{C}_{10} \mathrm{H}_{8}$ impurity probably overlapping the $8 \mathrm{~cm}^{-1}$ phonon (?) peak. Feature at $-16 \mathrm{~cm}^{-1}$ may be $8 \mathrm{~cm}^{-1}$ phonon (?) built on $\mathrm{C}_{10} \mathrm{H}_{8}$ origin. $\mathrm{cm}^{-1}$. The excitation source was a Coherent Radiation Laboratory Ar ion laser. The $4880 \AA$ laser emission, selected by a suitable interference filter, was used for the excitation.

\section{RESULTS AND DISCUSSION}

The results of the fluorescence $\left({ }^{1} B_{2 u} \rightarrow A_{\theta}\right)$ and phosphorescence $\left({ }^{3} B_{1 u} \rightarrow A_{q}\right)$ of naphthalene- $h_{8}$ guests in naphthalene- $d_{8}$ host are shown, respectively, in Figs. 1 and 2 . Figure 3 shows the phosphorescence of $2-\mathrm{DC}_{10} \mathrm{H}_{7}$ in $\mathrm{C}_{10} \mathrm{D}_{8}$. These figures display the sidebands built on the lower energy side of the pure electronic transition $(0-0$ band). A neutron-incoherent-scattering study of naphthalene- $h_{8}$ has been reported recently by Reynolds, Kjems, and White. ${ }^{8}$ For the sake of comparison, Fig. 4 displays our spectral results (at $2^{\circ} \mathrm{K}$ ) from the fluorescence and the phosphorescence of $\mathrm{C}_{10} \mathrm{H}_{8}$ in $\mathrm{C}_{10} \mathrm{D}_{8}$ along with the neutron-incoherent-scattering results of Reynolds et al. (at $120^{\circ} \mathrm{K}$ ) plotted at the same dispersion. Figure 5 compares the $\mathrm{C}_{10} \mathrm{H}_{8}$ in $\mathrm{C}_{10} \mathrm{D}_{8}$ phosphorescence sideband with a theoretical (semi-empirical) calculation by Pawley. ${ }^{7,9}$ Table I lists the frequencies of the peaks observed in these spectra.

First of all we would like to compare the results of the fluorescence and the phosphorescence. The sidebands in the low frequency region (up to about $60 \mathrm{~cm}^{-1}$ from the $0-0$ band) are very similar in the three emissions. The only exception is the sharp transition at 5 $\mathrm{cm}^{-1}$ in the phosphorescence spectrum of $\mathrm{C}_{10} \mathrm{H}_{8}$ in $\mathrm{C}_{10} \mathrm{D}_{8}$, which is absent in the fluorescence spectrum (and in the 2- $\mathrm{DC}_{10} \mathrm{H}_{7}$ in $\mathrm{C}_{10} \mathrm{D}_{8}$ phosphorescence spectrum). Its small width, compared to the other peaks in the sideband of the phosphorescence, combined with 


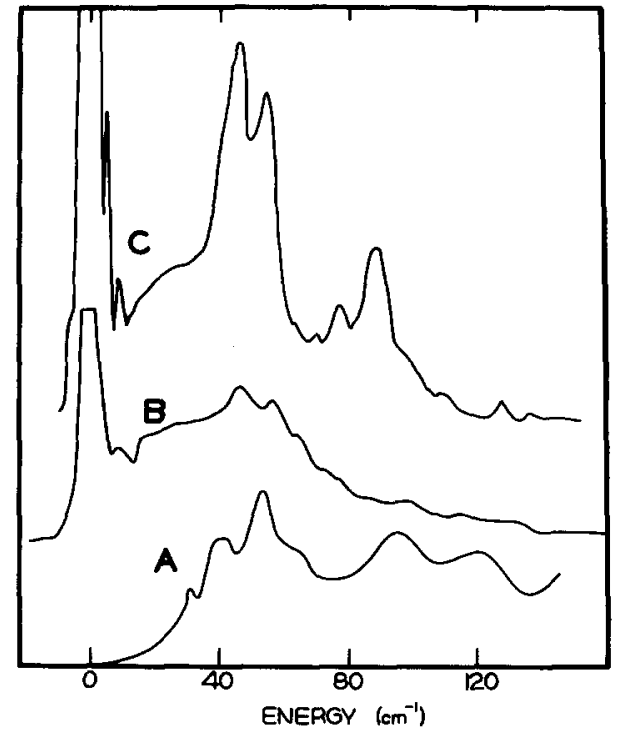

FIG. 4. This displays the phosphorescence and the fluorescence of naphthalene- $h_{8}$ in naphthalene- $d_{8}$ as host at $2^{\circ} \mathrm{K}$ (resolution of about $1 \mathrm{~cm}^{-1}$ ) together with the incoherent inelastic neutron scattering results at $120^{\circ} \mathrm{K}$ of Reynolds $e t a l .^{8} \mathrm{~A}$ is the incoherent neutron scattering result; $B$ and $C$ are, respectively, the fluorescence and the phosphorescence of naphthalene- $h_{8}$ (from Figs. 1 and 2).

the fact that it does not show up in the other two spectra, suggests that this transition is not part of the phonon spectrum but, perhaps, due to a trap. Although the peaks on the high frequency region $\left(>60 \mathrm{~cm}^{-1}\right)$ for the two emissions of $\mathrm{C}_{10} \mathrm{H}_{8}$ correlate in energy (as can be seen from Table I), the intensities with which they appear in these two emissions are drastically different. In our theoretical description this means that the amplitudes $C_{S, q}{ }^{f}$ for modes $S$ in the region above $60 \mathrm{~cm}^{-1}$ are very sensitive to the electronic state $f$. These phonon regions in naphthalene are dominated by librational motions. This implies that the guestlattice coupling (change in equilibrium configuration) along the librational coordinate in the ${ }^{1} B_{2 u} u^{1} A_{g}$ transition is very different from that in the ${ }^{3} B_{1 u}-{ }^{1} A_{0}$ transition. Furthermore, we see that the phonon sidebands in fluorescence appear with greater intensity relative to the zero phonon line than they do in phosphorescence, but we believe that this arises partly because of reabsorption at the fluorescence zero phonon line of the pure electronic transition. ${ }^{22}$ As the amplitudes $C_{S, q}{ }^{f}$ for mode $S, q$ are determined by the space group symmetry and not the molecular symmetry, one could expect them to be the same for the $S_{1}-S_{0}\left({ }^{1} B_{2 u}-\right.$ $\left.{ }^{1} A_{g}\right)$ and the $T-S_{0}\left({ }^{3} B_{1 u}-{ }^{1} A_{g}\right)$ transition of the same orbital nature. A possible source of such difference in $C_{S, q} f$ is that the singlet state of the guest is mixed with the host exciton band and as a result is delocalized to an extent of $10 \%$. Such delocalization (or mixing) with the host exciton band may induce significant change in $C_{S, q}$. This problem is treated in the Appendix. However, we admit that all these explanations are mere qualitative rationalizations. A quantitative understanding can be provided only by the detailed knowledge of the coefficient $C_{S, q}$.

Next we would like to compare the features of the phonon sideband in the emission spectra with those obtained by incoherent neutron scattering. Any correlation of peaks or critical point frequencies between our phonon sideband and the neutron incoherent scattering is dubious. However, we note that our spectra are obtained at $2^{\circ} \mathrm{K}$ whereas the neutron scattering results have been obtained at $120^{\circ} \mathrm{K}$. If we look into the results of Reynolds et al. ${ }^{8}$ we see that the neutron incoherent spectrum changes drastically from room temperature to $120^{\circ} \mathrm{K}$, and this change is not just in the frequencies of the critical points but also in the general features. It should be remembered that the inelastic neutron data suffer most of all from the fact that the "density of states" is actually heavily biased by hydrogen motions, and the exact weighting factor cannot easily be determined. Furthermore, multiphonon processes may significantly affect the data (there is no criterion to assess this, in contrast to the situation in our optical data, where the criterion of relative intensity of the $0-0$ to phonon sidebands can be used). We note that the theoretically calculated $^{7}$ hydrogen-amplitude-weighted density-of-states does not agree with the neutron data as well as the theoretical ${ }^{7,9}$ density-of-states agrees with our data (see below).

A significant advantage of the isotopic impurity sideband method is that various isotopic impurities can be used, like our use of $\mathrm{DC}_{10} \mathrm{H}_{7}$ in $\mathrm{C}_{10} \mathrm{D}_{8}$, in addition to $\mathrm{C}_{10} \mathrm{H}_{8}$, to check for consistency, both experimentally (i.e., eliminate spurious effects related to sample preparation) and theoretically (i.e., use energy denominator method to check on guest-host exciton mixing). Furthermore, vibronic origins can be compared to pure electronic ones (we used this to check on factors like reabsorption, defects, etc.). Finally, it

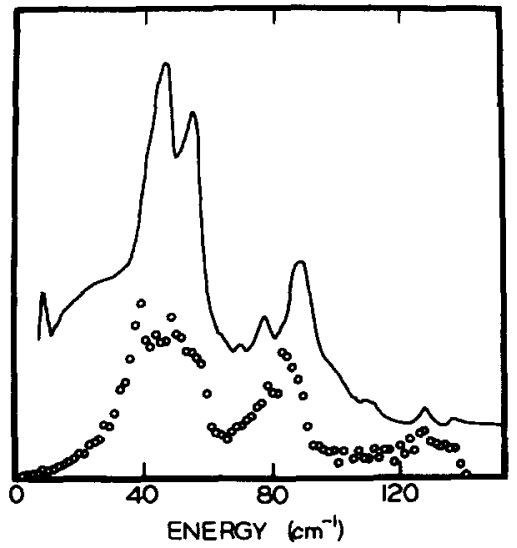

FIG. 5. This compares our naphthalene- $h_{8}$ in naphthalene-d $d_{8}$ phosphorescence result (solid curve) with the phonon densityof-states (curve made of circles) calculated theoretically by Pawley ${ }^{7,9}$ for $\mathrm{C}_{10} \mathrm{H}_{8}$ (see text and Table I). 
should be noted that we are observing the critical points in the phonon density-of-states of naphthalene- $d_{8}$ while the neutron values correspond to naphthalene- $h_{8}$, and that there is a relatively large uncertainty in the neutron scattering experiments with respect to both energy and intensity.

Comparing our phosphorescence sideband spectrum to the theoretical (semiempirical) atom-atom calculation by Pawley (Fig. 5), we find an excellent correspondence. Because of ease-of-reproduction we have taken Pawley's naphthalene- $h_{8}$ and not naphthalene- $d_{8}$ density-of-states display (The combined inaccuracy in calculation and reproductions justifies this-see Table I). It is hard to believe that the similarities displayed in Fig. 4 are all fortuitous. We also point out that the "spectral density" was not modified according to Eq. 13. While the correction factor $C_{S, q}$ is far from trivial we can make the qualitative statement that, at low energy, the major correction may be essentially that for the acoustical band of a one-molecule-per-cell cubic crystal, namely, $C_{S, q}^{f} \alpha\left(\omega_{S, q}\right)^{-1}$. It is obvious from Fig. 4 that such a correction would improve the agreement in the region $10-40 \mathrm{~cm}^{-1}$. We also note that while officially (see Table 1) we only count "peaks", there are other kinds of van Hove singularities present. However, we feel that we should not push our data too far at this stage. We just comment that the complete guest localization in the excited phosphorescent $\left({ }^{3} B_{2 u}\right)$ state and the low relative intensity of the phosphorescence sideband may indicate little dependence of $C_{S, q}{ }^{f}$ on $\omega$, thereby giving us a fairly faithful mapping of the density-of-phonon-states. On the other hand, the rigidlattice atom-atom calculation of $\mathrm{Pawley}^{7}$ is surprisingly successful.

Finally, we wish to present our results on the Raman spectra of naphthalene where a peak in the phonon density-of-states (not corresponding to $\mathbf{k}=0$ ) has been seen. Figure 6 shows the Raman spectrum of naphthalene- $d_{8}$ at $100^{\circ} \mathrm{K}$. The peak at about $40 \mathrm{~cm}^{-1}$ is, we believe, due to a peak in the phonon density-of-states at $100^{\circ} \mathrm{K}$. All other peaks except for this one in the phonon spectra of naphthalene have been observed before and explained as $\mathbf{k}=0$ transitions of the librational phonon bands. This $40 \mathrm{~cm}^{-1}$ peak appears with significant intensity only in the naphthalene- $d_{8}$ spectrum. In naphthalene- $h_{3}$ the corresponding peak is very weak and cannot be easily detected. Its appearance seems to depend on sample preparation. While one needs merely to say that any "defect" in the crystal will induce some spectral intensity near a density-of-states peak, we wish to point out that isotopic impurities have been shown capable of inducing such peaks, even in a situation very close to the amalgmation limit $\left({ }^{13} \mathrm{C}^{12} \mathrm{C}_{5} \mathrm{H}_{6}\right.$ in $\mathrm{C}_{6} \mathrm{H}_{6}$, in a very shallow trap situation)..$^{23}$ We therefore admit here that the natural abundance ${ }^{13} \mathrm{C}^{12} \mathrm{C}_{9} \mathrm{H}_{8}$ molecules (11\% mole concentration!) could be responsible for the weak band in $\mathrm{C}_{10} \mathrm{H}_{8}$, while in $\mathrm{C}_{10} \mathrm{D}_{8}$ the $\mathrm{C}_{10} \mathrm{D}_{7} \mathrm{H}$ impurities (about $8 \%$ mole) may be

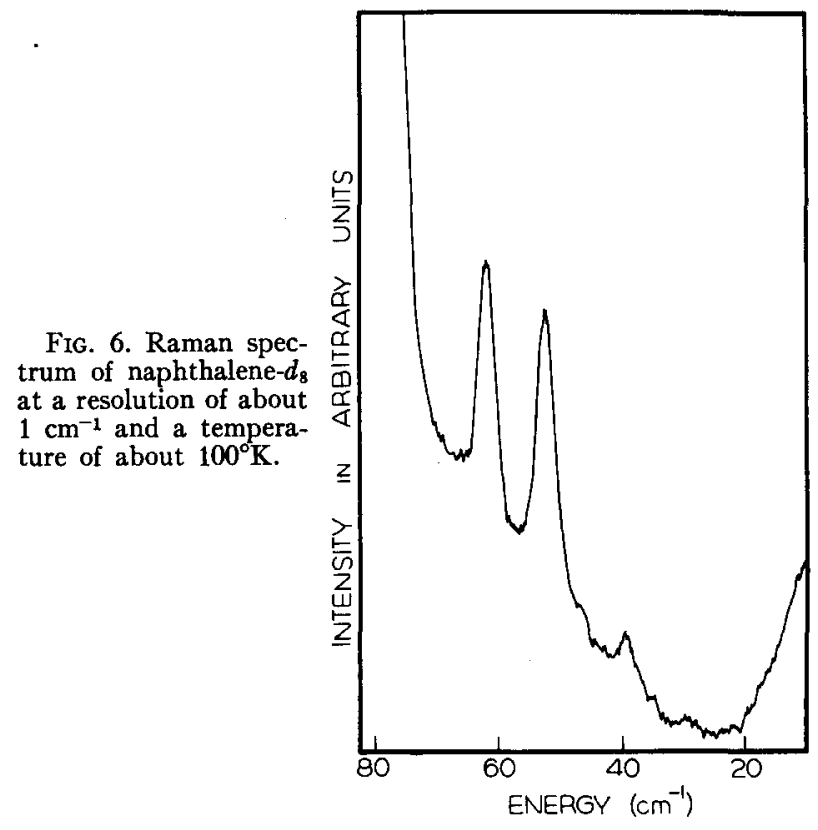

an even more potent perturber. Note that for both perturbers the absolute mass defect is the same $(\Delta m / m \cong$ $1 / 136$ ), but deuteration might also involve a forceconstant "defect". We note that the literature far infrared data by Hadni et al..$^{24,25}$ also indicate one (or more?) unexplained absorptions in the region of 40-55 $\mathrm{cm}^{-1}$, especially a broad unpolarized absorption peaked about $45 \mathrm{~cm}^{-1}$ at liquid nitrogen temperature (also one unassigned, but polarized, feature at about $115 \mathrm{~cm}^{-1}$ ). These features may have the same origin as our above mentioned Raman data. One value of the Raman data is in the comparison of the two kinds of doped crystal spectra-the direct observation of impurity (defect) induced host phonon spectra vs the indirect method, i.e., sidebands on guest localized electronic transitions. It is obvious that the low concentration $(0.25 \%)$ indirect spectra are much more powerful than the high concentration (10\% or more) direct ones. They are not only much easier to obtain but give a far more faithful mapping of the host phonon density-of-states. They also do not depend on sample preparation.

In principle, phonon sidebands of vibrational and vibronic excitons can be utilized the same way as those of electronic excitons. This way one could study $\mathrm{C}_{10} \mathrm{H}_{8}$ as well as $\mathrm{C}_{10} \mathrm{D}_{8}$ phonons. With very narrow exciton bands neat crystal studies may suffice. While Raman studies will be limited by the same resolution as ultraviolet ones, with an added intensity problem, infrared studies might be ideal, provided that the spectral region is not overcrowded. We are presently looking into it.

The chief value of mapping the phonon singularities by the guest-exciton-to-host-phonon transition is in its apparent unbiased faithfullness to the singularities of the phonon density-of-states. Model calculations, like Pawley's, ${ }^{9}$ can now be easily calibrated on these singularities. The result will be both an improved model (like 
improvements on the atom-atom potential) and an improved phonon density-of-states. The uses of the latter are obvious: improved calculated thermodynamic properties, like heat capacity, and improved calculated energy transport properties, from thermal conductivity to radiationless transitions and radiation chemistry. Specifically for naphthalene, our results give added credibility to Pawley's calculated thermodynamic functions (Debye $\theta$ 's as function of temperature) ${ }^{9}$

\section{CONCLUSION}

A new optical method of mapping the phonon densityof-states in molecular solids has been demonstrated. Small, polycrystalline, and relatively impure samples can be used now to give a phonon energy distribution to an accuracy of $1 \mathrm{~cm}^{-1}$ or better. Coupled with simple model calculations, this method is expected to give very detailed density-of-states functions, as well as an improved theoretical understanding of potentials in molecular crystals.

\section{APPENDIX}

In this appendix we treat the effect of guest-host mixing on the phonon sideband in the electronic transition of the guest. The Hamiltonian for the crystal containing an impurity is defined as

$$
B=H_{\mathrm{el}}+H_{\mathrm{ph}}+H_{\mathrm{el}-\mathrm{ph}}
$$

where $H_{\mathrm{el}}$ is the pure electronic Hamiltonian given as

$$
\begin{aligned}
H_{\mathrm{el}}{ }^{f}=\sum_{n \alpha}\left[E^{f}\right. & \left.+D_{n \alpha}{ }^{f}(0)\right] a_{n \alpha}{ }^{+} a_{n \alpha} \\
& +\sum_{n \alpha, m \beta}\left[M_{n \alpha, m \beta}+V_{n \alpha, m \beta}\right] a_{n \alpha}{ }^{+} a_{m \beta} .
\end{aligned}
$$

Here $E^{f}$ is the zero order excitation energy of the host molecules corresponding to the excited state $f . D_{n \alpha} f(0)$ is the static solvent shift. $M_{n \alpha, m \beta}$ is the excitation exchange interaction of the host molecules and $V_{n \alpha, m \beta}$ is the perturbation due to the guest (do not confuse with $V$ used before). In Eq. (A1),

$$
H_{\mathrm{ph}}=\sum_{s, q} \omega_{s, q}\left[b_{s, q}+b_{s, q}+\frac{1}{2}\right] .
$$

$\boldsymbol{H}_{\mathrm{el-ph}}$ is the electron-phonon interaction arising from the change in the equilibrium position of the molecule during its electronic excitation. It is given as

$$
B_{\mathrm{el}-\mathrm{ph}}=\sum_{n \alpha} \sum_{m \beta}\left(\partial D_{n \alpha} \delta / \partial R_{u m \beta}\right)_{0} R_{u m \beta} a_{n \alpha}{ }^{+} a_{n \alpha} .
$$

The Hamiltonian (A2) cannot be diagonalized in the presence of (A4). At this point it becomes necessary to define the limits of strong coupling and weak coupling. In analogy to the definition of Simpson and Peterson $^{26}$ we define the strong coupling between the guest and the host to be the case where the local lattice relaxation $H_{\mathrm{el}-\mathrm{ph}}$ is weaker than $M_{n \alpha, m \beta}$. We feel that our case represents this limit as the intensity in the phonon band is small and multiphonon effects are seen to be minimal. In this case we can diagonalize the Hamiltonian (A2) and treat (A4) as a perturbation on the Hamiltonian (A2). The Hamiltonian is diagonalized by making a canonical transformation of the operators $a_{n \alpha}{ }^{+}$and $a_{n \alpha}$ as

$$
a_{n \alpha}=\sum_{p} U_{p n \alpha} a_{p}
$$

and

$$
a_{n \alpha}{ }^{+}=\sum_{p} U_{p n \alpha}^{*} a_{p}{ }^{+},
$$

where $a_{p}{ }^{+}$and $a_{p}$ are the operators for the eigenstate " $p$ " of the Hamiltonian (A2). The transformation coefficients $U_{p n \alpha}$ obey the following normalization condition

$$
\sum_{n \alpha} U_{p n a}{ }^{*} U_{p^{\prime} n a}=\delta_{p p^{\prime}}, \sum_{p} U_{p n \alpha}{ }^{*} U_{p n^{\prime} \alpha^{\prime}}=\delta_{n n}, \delta_{\alpha \alpha^{\prime}} .
$$

This problem of diagonalization is discussed by Izyumov ${ }^{27}$ and by Sommer and Jortner. ${ }^{28}$ In our case the guest is an isotopic impurity and we assume that the perturbation due to it involves only the trap depth at the guest site $o \alpha_{1}$ and no change in excitation exchange. This means

$$
V_{n \alpha, m \beta}=V_{0} \delta_{n \alpha, \alpha \alpha} \delta_{m \beta, o \alpha 1} .
$$

For this case the solution for the transformation matrix yields ${ }^{27,28}$

$$
U_{p n \alpha}=G_{n \alpha, o \alpha_{1}}{ }^{0}\left(E_{p}\right)\left\{\left(-d / d E_{p}\right) G_{0}\left(E_{p}\right)\right\}^{-1 / 2}
$$

where $G_{n \alpha, o \alpha_{1}}{ }^{0}\left(E_{p}\right)$ is the Green's function of the neat crystal in the localized representation and is given as

$$
\begin{aligned}
G_{n \alpha, o \alpha_{1}}{ }^{0}\left(E_{y}\right)=N^{-1} \sum_{k, j}\left\{A_{\alpha_{1}} j^{*} A_{\alpha j} /\left[E_{p}-E_{j}(\mathbf{k})\right]\right\} \\
\times \exp \left[i \mathbf{k} \cdot\left(\mathbf{x}_{o \alpha_{1}}-\mathbf{x}_{n \alpha}\right)\right],
\end{aligned}
$$

where $j$ labels the interchange group states in the neat host crystals and $E_{j}(\mathbf{k})$ gives the exciton dispersion of the host band. $A_{\alpha j}$ is the interchange group transformation coefficient. Also,

$$
G_{0}\left(E_{p}\right)=G_{o \alpha_{1}, o \alpha_{1}}{ }^{0}\left(E_{p}\right) .
$$

After diagonalizing the Hamiltonian (A2) let us return to $H_{\mathrm{el}-\mathrm{ph}}$. Expansion for $R_{u m \beta}$ in terms of phonon creation and annihilation operators yields

$$
\begin{aligned}
& H_{\mathrm{el}-\mathrm{ph}}=\sum_{n \alpha} \sum_{S, q}\left(2 M N \omega_{S, q}\right)^{-1 / 2} \\
& \times \sum_{l, \beta, u}\left\{\left(\partial D_{n \alpha,(n+l) \beta^{f}} / \partial R_{u n \alpha}\right)_{0} \eta_{\alpha}{ }^{u}(S, q)\right. \\
& \left.+\left(\partial D_{n \alpha,(n+l) \beta^{f}} / \partial R_{u(n+l) \beta}\right)_{0 \eta \beta^{u}}(S, q) \exp (i q \cdot 1)\right\} \\
& \times \exp \left(i \mathrm{q} \cdot \mathbf{x}_{n}\right)\left[b_{S, q}+b_{S,-q}{ }^{+}\right] a_{n \alpha}{ }^{+} a_{n \alpha}
\end{aligned}
$$

where the sums $1, \beta$ run over the near neighbors of the molecule at $n \alpha$. 
Let us define $L_{S, q}{ }^{f}(\alpha)$ as

$$
\begin{aligned}
& L_{S, q}{ }^{f}(\alpha)=\sum_{l, \beta, u}\left\{\left(\partial D_{n \alpha,(n+l) \beta^{f}} / \partial R_{u n \alpha}\right)_{0} \eta_{\alpha}{ }^{u}(S, q)\right. \\
& \left.+\left(\partial D_{n, \alpha,(n+l) \beta^{f}} / \partial R_{u(n+l) \beta}\right)_{0} \eta_{\beta}{ }^{u}(S, q) \exp (i \mathrm{q} \cdot 1)\right\} .
\end{aligned}
$$

Making use of Eqs. (A5), (A7) and (A10), equation (A9) yields:

$$
H_{\mathrm{el}-\mathrm{ph}}=N^{-1 / 2} \sum_{p, p^{\prime}} D_{S, q^{f}}\left(p, p^{\prime}\right)\left[b_{S, q}+b_{S,-q^{+}}{ }^{+}\right] a_{p}{ }^{+} a_{p^{\prime}},
$$

where

$$
\begin{aligned}
& D_{S, q}{ }^{f}\left(p, p^{\prime}\right)=\sum_{n \alpha}\left(2 M \omega_{S, q}\right)^{-1 / 2} L_{S, q}{ }^{f}(\alpha) \\
& \quad \times \exp \left(i \mathbf{q} \cdot \mathbf{x}_{n}\right) G_{n \alpha, o \alpha_{1}}{ }^{0}\left(E_{p}\right) \\
& \times G_{o \alpha_{1}, n \alpha^{0}}\left(E_{p^{\prime}}\right)\left[-\left(d / d E_{p}\right) G_{0}\left(E_{p}\right)\right]^{-1 / 2} \\
& \quad \times\left[-\left(d / d E_{p^{\prime}}\right) G_{0}\left(E_{p^{\prime}}\right)\right]^{-1 / 2} . \quad(\mathrm{A} 12) \\
& D_{S, q^{f}}\left(p, p^{\prime}\right)=\sum_{j} \sum_{j^{\prime}} N^{-1} \sum_{K} \sum_{\alpha}\left(2 M \omega_{S, q}\right)^{-1 / 2} L_{S, q^{\prime}}(\alpha) \\
& \times \frac{A_{\alpha_{j} j} A_{\alpha_{1} j^{\prime}}, A_{\alpha^{\prime}}{ }^{*} A_{\alpha j^{\prime}}}{\left\{E_{p}-E_{j}(\mathbf{k})\right\}\left\{E_{p^{\prime}}-E_{j^{\prime}}(\mathbf{k}+\mathbf{q})\right\}} \exp \left(-i \mathbf{q} \cdot \mathbf{x}_{\alpha-\alpha_{1}}\right) \\
& \quad \times\left\{-\left(d / d E_{p}\right) G_{0}\left(E_{p}\right)\right\}^{-1 / 2}\left\{-\left(d / d E_{p^{\prime}}\right) G_{0}\left(E_{p^{\prime}}\right)\right\}^{-1 / 2} .
\end{aligned}
$$

Let us suppose that the eigenstate associated with the guest is $p_{1}$. The diagonal term $D_{S, q}{ }^{f}\left(p_{1}, p_{1}\right)$ of equation (A11) is the Franck-Condon effect in the guest electronic state and is equivalent to the coupling function $D_{z, q}^{f}$ of a localized guest electronic state which has been discussed earlier. It can be seen from the expression for $D_{s, q}\left(p_{1}, p_{1}\right)$ given by equation (A13) that it depends on the exciton dispersion and may show characteristics that are significantly different from those of the corresponding localized guest state coupling function $D_{s, q}$. Such difference will lead to a renormalization in the intensity pattern of the various peaks in the phonon sideband when the guest-host mixing becomes appreciable. This is because the functions $D_{8, q}{ }^{f}$ determine the amplitudes $C_{s, q} f$ and, thus, the relative intensity of the phonon peaks.

We also see that the interaction Hamiltonian (A11) contains the off-diagonal term $D_{s, q} f\left(p_{1}, p\right)$ in which $p_{1} \neq p$. This interaction mixes the guest state $p_{1}$ with other states $p$ which correspond mainly to host states (the exciton band) and is, thus, a Herzberg-Teller type interaction. In the singlet state of naphthalene where the guest site is only about $40 \mathrm{~cm}^{-1}$ below the $160 \mathrm{~cm}^{-1}$ wide host exciton band, this mixing can be significant. As the diagonal interaction (the FranckCondon interaction) is stronger than the off-diagonal Herzberg-Teller type interaction, we would include the effect of the diagonal interaction by a canonical transformation described earlier, while the HerzbergTeller interaction will be treated by first order perturbation theory. The transition moment for the one phonon transition $p_{1}, 0_{s, q} \rightarrow g, 1_{s, q}$ is given as ${ }^{11}$

$$
\begin{aligned}
& \mu\left(p_{1}, 0_{S, q} \rightarrow g, 1_{S, q}\right) \\
&=\mu^{0}\left(p_{1}, 0_{S, q} \rightarrow g, 0_{S, q}\right)\left[D_{S, q} f\left(p_{1}, p_{1}\right) / \omega_{S, q}\right] \\
& \quad+\sum_{p \neq p_{1}} \boldsymbol{\mu}^{0}\left(p, 0_{S, q} \rightarrow g, 0_{S, q}\right) \\
& \times\left[D_{S, q} f\left(p, p_{1}\right) /\left(E_{p}+\omega_{S, q}-E_{p_{1}}\right)\right] .
\end{aligned}
$$

The intensity expression, thus, contains three terms: (i) the guest intensity term involving $\mid \mu^{0}\left(p_{1}, 0_{S, q} \rightarrow\right.$ $\left.g, 0_{S, q}\right)\left.\right|^{2}$; (ii) the intensity term $\left|\boldsymbol{\mu}^{0}\left(p, 0_{\mathcal{S}, q} \rightarrow g, 0_{S, q}\right)\right|^{2}$ borrowed from the host band and (iii) the interference term $\left|\mu^{0}\left(p_{1}, 0_{S, q} \rightarrow g, 0_{S, q}\right) \cdot \mu^{0}\left(p, 0_{S, q} \rightarrow g, 0_{S, q}\right)\right|$. The interference term which involves the dot product of two vectors can be important in determining the intensity of a particular phonon transition.

The Herzberg-Teller interaction $D_{S, q} f\left(p_{1}, p\right)$ gives rise to the quadratic electron-phonon interaction in the second order. This quadratic interaction also has an effect of renormalizing the phonon transition intensity. This problem has been discussed recently by Small. ${ }^{29}$

To conclude, we have shown that the guest-host mixing can manifest itself as a significant change in the intensity characteristics of the phonon sideband.

* Supported by NSF Grant GP-18718 and NIH Grant NS08116. 1 A. B. Zahlan (ed)., Excitons, Magnons and Phonons in Molecular Crystals (Cambridge U. P., New York, 1968)

${ }^{2} \mathrm{H}$. Boutin and S. Yip, Molecular Spectroscopy with Neutrons (MIT Press, Cambridge, Mass., 1968).

3 T. Kitagawa, Appl. Spectrosc. Rev. 6, 79 (1972).

4 G. Dolling and B. M. Powell, Proc. Roy. Soc. (London) A319, 209 (1970); R. Stockmeyer and H. Stiller, Phys. Status Solidi 27, 269 (1968).

${ }^{5}$ G. S. Pawley and E. A. Yeats, Solid State Commun. 7, 385 (1969).

${ }^{6}$ V. A. Lutz and W. Halg, Solid State Commun. 8, 165 (1970).

7 G. S. Pawley, P. A. Reynolds, J. K. Kjems, and J. W. White, Solid State Commun. 9, 1353 (1971).

${ }^{8}$ P. A. Reynolds, J. K. Kjems, and J. W. White, J. Chem. Phys. 56, 2928 (1972).

${ }^{9}$ G. S. Pawley, Phys. Status Solidi 20, 347 (1967)

${ }_{10}$ G. W. Robinson, Ann. Rev. Phys. Chem. 21, 429 (1970).

${ }^{11}$ R. M. Hochstrasser and P. N. Prasad, in Excited States Vol. 1, edited by E. C. Lim (Academic, New York, to be published).

${ }^{12}$ A. S. Davydov, Theory of Molecular Excitons, translated by B. Dresner (Plenum, New York, 1971).

${ }^{13}$ S. Fisher and S. A. Rice, J. Chem. Phys. 52, 2089 (1970).

${ }^{14}$ M. R. Grover and R. Silbey, J. Chem. Phys. 52, 2099 (1970).

${ }^{15}$ C. Mavroyannis, J. Math. Phys. 11, 491 (1970).

${ }^{16}$ R. M. Hochstrasser and P. N. Prasad, J. Chem. Phys. 56, 2814 (1972)

${ }_{17}$ P. H. Chereson, P. S. Friedman, and R. Kopelman, J. Chem. Phys. 56, 3716 (1972).

${ }_{18}$ P. N. Prasad and R. Kopelman, J. Chem. Phys. 57, 863 (1972)

${ }^{19}$ Y. Onodera and Y. Toyozawa, J. Phys. Soc. Japan 24, 341 (1968).

${ }_{20} \mathrm{~J}$. Hoshen, Ph.D. thesis, Tel-Aviv University, 1971; J. Hoshen and J. Jortner, J. Chem. Phys. 56, 933 (1972).

${ }_{21}$ G. Venkataraman and V. C. Sahni, Rev. Mod. Phys. 24, 409 (1970). 
${ }^{22}$ This conclusion is based on comparison with the phonon side-bands of vibronic progressions.

${ }^{23} \mathrm{~J}$. C. Laufer and R. Kopelman, J. Chem. Phys. 57, 3202 (1972).

${ }_{24}$ A. Hadni, in Excitons, Magnons and Phonons in Molecular Crystals, edited by A. B. Zahlan (Cambridge U. P., New York, $1968)$, p. 31.
${ }^{25}$ A. Hadni, B. Wyncke, G. Morlot, and X. Gerbaux, J. Chem. Phys. 51, 3514 (1969).

${ }^{26} \mathrm{~W}$. T. Simpson and D. L. Peterson, J. Chem. Phys. 26, 588 (1957)

${ }_{27}$ Y. A. Izyumov, Advan. Phys. 14, 569 (1965)

${ }^{28}$ B. S. Sommer and J. Jortner, J. Chem. Phys. 50, 187 (1969).

${ }_{28}$ G. J. Small, Chem. Phys. Letters 15, 147 (1972).

\title{
Role of Time Delay in Chemical Reaction Rates
}

\author{
Rutger A. van Santen* $\dagger$ \\ Stanford Research Institute, Menlo Park, California 94025
}

(Received 2 June 1972)

\begin{abstract}
Using the concept of resonant states, the expressions for the rate of association and dissociation are derived. A density matrix formalism is developed, which enables us to discuss the conditions for which conventional expressions of the reaction rates are valid. It is shown that an Arrhenius type expression is only valid if the widths of the resonant states are larger than $\pi k T$, which implies that the decay of resonant states is independent of the way they are formed. As long as this condition and the steady state assumption holds for the concentration of molecules in resonant states, the interference effects of overlapping resonances are found to vanish. The connection with transition state theory is derived. General new expressions valid for all pressures are presented. The role of time delay in them is discussed.
\end{abstract}

\section{INTRODUCTION}

Recently, new developments in scattering theory, which had their origin in nuclear physics, have been applied to the development of expressions for chemical reaction rates. The chemical reaction is considered to proceed via the intermediate of a transient collision complex, which after some time breaks down into products.

The theory of resonant scattering has been developed to describe this type of collision, therefore, it seemed appropriate to study its consequences in the theory of chemical reaction rates. The work of Mies and Krauss, ${ }^{1}$ Mies, ${ }^{2}$ Rice, ${ }^{3}$ and Levine ${ }^{4}$ provide some outstanding examples of it.

Mies and Krauss, dealing with high pressure unimolecular rate constants, showed that those rates can be expressed in the time delay of the reverse scattering process. This is because the rates are found to be proportional to the density of resonant states averaged over a Boltzmann distribution. The time delay is defined as the difference in time two particles need to travel a particular distance, when collisions are present and when they are not present. ${ }^{5}$ It is found that the time delay $Q$ and the density of states $\rho$ have a very simple relationship ${ }^{6}$ :

$$
\rho=(1 / h) Q .
$$

This explains the occurrence of time delay in the calculations of Mies and Krauss.

In 1962, Smith ${ }^{7}$ presented a formula for the rate of association of two particles by collisions with a third inert body. He found that this rate will depend on the time delay of the two particles that are going to associ- ate. It will be shown that this result, however, is only valid at low pressures.

In this paper we extend those results to the high pressure case and therefore are able to connect it with the results of Mies and Krauss. The general result is a new formula, which is valid for all pressures.

One of the crucial assumptions in the theory of unimolecular reactions is the existence of a timegap between the moment a molecule is activated and the instant the molecule will decompose after redistribution of its internal energy. ${ }^{8}$ One can wonder whether this timegap is equal to the time delay of the reverse process. This appears not to be true, but an intimate relationship exists.

Rice ${ }^{9}$ found that the rate of dissociation of a molecule depends on an average time the activated molecule exists.

We will provide a unification of the theories that are related to classical theories of chemical reaction rates, like the transition state theory, with those that are based on resonant scattering theories.

Expressions connecting the cross section of a two body rearrangement collision with reaction rates were presented in the early 1960 's. ${ }^{7,10,11}$ They are essentially a Boltzmann energy average over the cross section and are based on the Wang-Chang and Uhlenbeck Boltzmann equation. ${ }^{12}$ This equation was developed to derive the transport properties of a system of particles in which inelastic collisions can occur. If $T_{i \rightarrow f}$ is the transition matrix for scattering from initial state $i$ to final state $f$, the expression for the rate becomes

$$
k_{i \rightarrow f}=\frac{2 \pi}{\hbar} Z^{-1} \int_{0}^{\infty} d E \exp (-E / k T)\left|T_{i \rightarrow f}\right|^{2} .
$$

